\section{Brachyury establishes the embryonic mesodermal progenitor niche}

\author{
Benjamin L. Martin and David Kimelman ${ }^{1}$ \\ Department of Biochemistry, University of Washington, Seattle, \\ Washington 98195, USA
}

Formation of the early vertebrate embryo depends on a Brachyury/Wnt autoregulatory loop within the posterior mesodermal progenitors. We show that exogenous retinoic acid (RA), which dramatically truncates the embryo, represses expression of the zebrafish brachyury ortholog no tail (ntl), causing a failure to sustain the loop. We found that $\mathrm{Ntl}$ functions normally to protect the autoregulatory loop from endogenous RA by directly activating cyp26a1 expression. Thus, the embryonic mesodermal progenitors uniquely establish their own niche-with Brachyury being essential for creating a domain of high Wnt and low RA signaling — rather than having a niche created by separate support cells.

Supplemental material is available for this article.

Received June 23, 2010; revised version accepted November 1, 2010.

Stem and progenitor cells play an essential role during the formation of the vertebrate body and the subsequent homeostasis of the adult form. A growing body of work has demonstrated that stem/progenitor cells exist throughout the embryo and adult in niches-unique physical and molecular environments that support the long-term maintenance of these populations (Morrison and Spradling 2008; Voog and Jones 2010). During vertebrate development, a population of mesodermal progenitor cells residing in the tailbud continuously releases differentiating cells throughout somitogenesis (Dubrulle and Pourquie 2004). We showed previously that the maintenance of this population is dependent on an autoregulatory loop between the T-box transcription factor Brachyury and canonical Wnt signaling, with a failure of this loop resulting in a truncated body axis (Martin and Kimelman 2008), explaining why Brachyury plays such a major role in vertebrate development (Martin and Kimelman 2009).

Retinoic acid (RA) signaling is another factor that can affect the formation of the body. Addition of RA to vertebrate embryos causes severe posterior truncations, superficially similar to a loss of Brachyury or Wnt signaling (Sive et al. 1990). Here we show that RA blocks the function of the autoregulatory loop by inhibiting no tail (ntl) expression, resulting in a failure to sustain Wnt signaling. We found that $\mathrm{Ntl}$ protects the mesodermal progenitors from RA produced in the adjacent somites by directly

[Keywords: Brachyury; no tail; Wnt; retinoic acid; mesoderm; niche] ${ }^{1}$ Corresponding author.

E-MAIL kimelman@uw.edu; FAX (206) 616-8676.

Article is online at http://www.genesdev.org/cgi/doi/10.1101/gad.1962910. activating the expression of the RA-metabolizing enzyme Cyp26a1. Finally, we show that individual mesodermal progenitor cells do not need to express $n t l$, wnts, or cyp26a1 as long as their neighbors express these genes, demonstrating that the essential role of Brachyury and its downstream targets is to create the niche in which the mesodermal progenitors are maintained in a progenitor state throughout somitogenesis.

Previously identified stem/progenitor cell niches are established by a separate population of niche support cells that exist adjacent to the stem/progenitor cells. The embryonic mesodermal progenitor population that we describe is unique in its ability to serve as both the niche support cells and the progenitor cells, without subfunctionalization into two separate cell types. These results have significant implications in understanding the pathogenesis of Brachyury-expressing human cancers.

\section{Results and Discussion}

\section{$R A$ disrupts posterior growth by inhibiting $\mathrm{ntl}$ expression}

RA has been known for many years to cause severe posterior truncations in vertebrate embryos exposed during gastrulation (Murakami and Kameyama 1965; Sive et al. 1990; Kessel and Gruss 1991; Shum et al. 1999|. We found that this sensitivity persists throughout most of somitogenesis when the posterior half of the embryo is forming (Fig. 1B; data not shown), demonstrating that the posterior truncation caused by RA treatment is not the result of early patterning defects. We therefore investigated the Brachyury-Wnt autoregulatory loop, which is necessary for maintaining the mesodermal progenitors of the tailbud (Martin and Kimelman 2008, 2009). To determine if RA directly interferes with the maintenance of this signaling loop, we examined the expression of $n t l$ and $w n t 3 a$. After just $1 \mathrm{~h}$ of RA treatment, $n t l$ expression is completely lost in the mesodermal progenitors of the tailbud $100 \%$ of RA-treated embryos exhibited reduction in $n t l$ expression, $N=15$ ) (Fig. $1 \mathrm{C}, \mathrm{D})$, whereas wnt3a expression is normal (100\% of RA-treated embryos exhibited normal wnt $3 a$ expression, $N=7$ ) (Fig. 1E,F). After $2 \mathrm{~h}$ of RA exposure, wnt3a expression is down-regulated in the mesodermal progenitors $(100 \%$ of RA-treated embryos exhibited reduced wnt3a expression, $N=7$ ) (Fig. 1G,H). These results indicate that RA signaling impinges on the maintenance of the Brachyury-Wnt autoregulatory loop by first repressing $n t l$ expression, which leads to a loss of wnt expression and a disruption in posterior growth.

While the addition of RA efficiently represses ntl expression in mesodermal progenitors, it has little effect on $n t l$ expression in axial mesoderm during both the gastrula and tailbud stages (Fig. 1D; Supplemental Fig. S1). The lack of $n t l$ repression in axial mesoderm was determined to be due to the lack of RA receptor (RAR) expression in this region. While four RARs are expressed in the tailbud, there is a clear absence of RAR expression in zebrafish axial mesoderm (Thisse and Thisse 2008), suggesting that exogenous RA cannot repress $n t l$ in axial mesoderm due to a lack of receptor expression. Indeed, misexpression of RAR in the axial mesoderm led to RA-induced repression of $n t l$ in this tissue (Supplemental Fig. S1). We further 


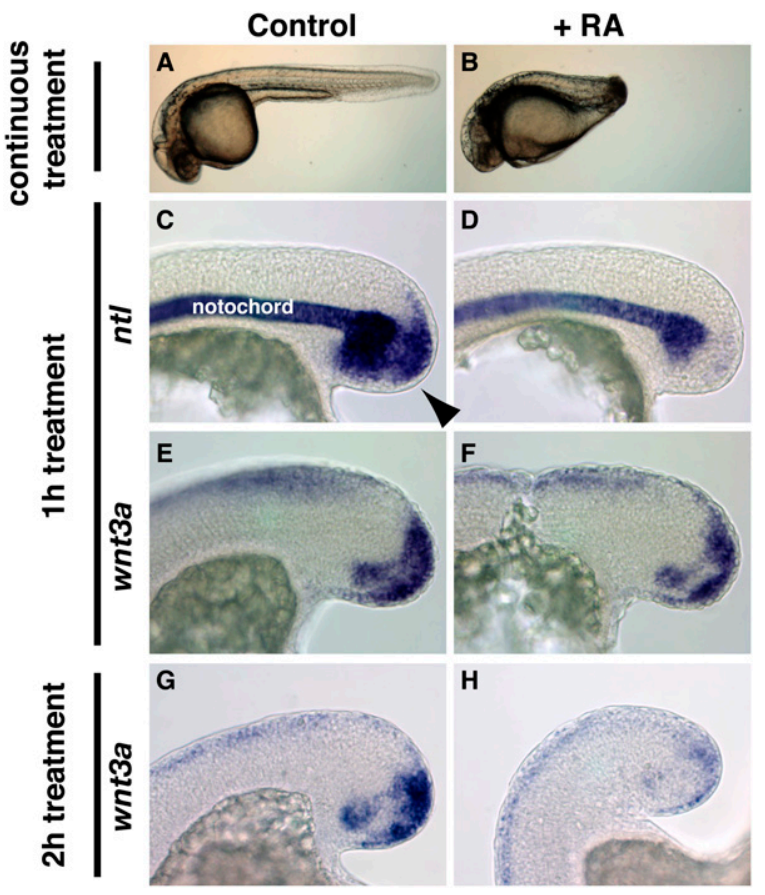

Figure 1. RA impinges on the $\mathrm{Ntl}-\mathrm{Wnt}$ autoregulatory loop by repressing $n t$ l expression. $(A, B)$ Embryos (36 h post-fertilization [hpf]) treated with vehicle alone $(A)$ or $1 \mu \mathrm{M}$ RA $(B)$, beginning at bud stage. (B) RA treatment causes severe posterior truncation. ntl $(C, D)$ and wnt $3 a(E, F)$ expression after $1 \mathrm{~h}$ treatment with vehicle $(C, E)$ or RA $(D, F)$, beginning at the 12 -somite stage. Within $1 \mathrm{~h}$, mesodermal progenitor (arrowhead in $C$ ) ntl expression is lost $(D)$, whereas wnt3a expression is normal $(F)$. After $2 \mathrm{~h}$ of RA treatment, wnt3a expression is lost from the mesodermal progenitors $(H)$ as compared with vehicle treatment $(G)$. $(D)$ Note that the expression of $n t l$ in the notochord is not affected by RA treatment. $(\mathrm{C}-\mathrm{H})$ Lateral view of the posterior end.

investigated the mechanism of RA-induced repression of $n t l$ and discovered that RA/RAR likely acts indirectly through the activation of an unknown $n t l$ repressor (Supplemental Fig. S2).

\section{Ntl protects mesodermal progenitors from $R A$ by regulating cyp26a1 expression}

During normal development, RA is produced in the posterior somites, where it plays an important role in ensuring that the somites form symmetrically (Kawakami et al. 2005; Vermot and Pourquie 2005; Sirbu and Duester 2006). The production of RA is controlled by the localized expression of an aldehyde dehydrogenase (aldh1a2) (Niederreither et al. 1997; Dobbs-McAuliffe et al. 2004), whereas the posterior end of the embryo expresses the RA-metabolizing enzyme cyp26a1 (Abu-Abed et al. 2001; Sakai et al. 2001; Emoto et al. 2005). The expression of cyp26a1 is directly activated by RA signaling, but the posterior tailbud expression domain is independent of this regulation, indicating that another factor regulates posterior activation (Hu et al. 2008). Since $n t l$ and cyp26a1 are both expressed in the mesodermal progenitors, and since exogenous RA is capable of repressing $n t l$, we tested the hypothesis that $\mathrm{Ntl}$ protects its own expression by regulating cyp26a1. In $n t l$ mutant embryos at a stage before defects in somite formation are observed, cyp26a1 expression is completely lost from the mesodermal progenitors (100\% of $n t l$ mutants affected, $N=6)$ (Fig. 2A,B). In contrast, embryos mutant for the tailbud-specific T-box transcription factor spt showed no reduction in cyp26a1 (100\% with strong cyp26a1 expression, $N=20$ ) (Fig. 2C,D). The expanded cyp26a1 domain is due to the failure of $s p t$ mutant cells to complete differentiation (Griffin and Kimelman 2002). These results indicate that $\mathrm{Ntl}$ is specifically necessary for cyp26a1 expression in the mesodermal progenitors.

In order to determine whether $\mathrm{Ntl}$ is sufficient for cyp26a1 expression during the posterior growth phase, we generated a transgenic line with the $n t l$ coding region under the control of a synthetic heat-shock promoter. Heat-shock activation of $n t l$ caused ubiquitous expression of cyp26a1 throughout the embryo, demonstrating that $\mathrm{Ntl}$ is sufficient for cyp26a1 expression (100\% of transgenic embryos had ubiquitous cyp26a1 expression $[N=30]$, whereas cyp26a1 expression was normal in all nontransgenic siblings $[N=17])$ (Fig. 2E,F). Together, these results indicate that $\mathrm{Ntl}$ protects its own expression and the mesodermal progenitor population from the effects of endogenous somite-produced RA by activating cyp26a1 expression.

In mice, posterior cyp26a1 expression is lost when Fgfr1 is conditionally mutated in all brachyury-expressing mesodermal cells (Wahl et al. 2007). This and experiments in chicks showing that RA inhibits Fgf signaling (Diez del Corral et al. 2003) raised the possibility that Ntl regulates cyp26a1 expression indirectly through activation of Fgf signaling. In contrast, our previous work demonstrated that Fgf signaling is maintained in mesodermal progenitors lacking $\mathrm{Ntl}$ function (Martin and Kimelman 2008), suggesting that Ntl regulation of cyp26a1 is not through Fgf activation. We determined that Fgf signaling is not involved in the posterior regulation of cyp26a1 using a heat-shock-inducible transgenic line expressing dominant-negative Fgfrl from the hsp 70 promoter (HS:dnfgfr 1 ) (Lee et al. 2005). We used this line to inhibit Fgf signaling after the end of gastrulation (bud stage) so that we could bypass the early gastrula requirement of Fgf for all posterior mesoderm formation in zebrafish (Griffin et al. 1995). Despite complete loss of expression of the direct Fgf targets pea3 and erm after heat-shock activation of $\operatorname{dnfgfr} 1$ (100\% of transgenic embryos had complete pea3 and erm loss, $N=20$ and $N=19$, respectively) (Fig. 2G-J), cyp26a1 expression in the posterior tailbud was normal $(100 \%$ of transgenic embryos had normal cyp26a1 expression, $N=$ 19) (Fig. 2K,L). Correspondingly, we also found that $n t$ l expression was normal in the tailbud after Fgf loss of function $(100 \%$ of transgenic embryos had normal $n t l$ expression, $N=20$ ) (Fig. 2M,N). These data confirmed that zebrafish cyp26a1 is not regulated by Fgf signaling, and raised the possibility that Ntl directly activates cyp26a1 expression in mesodermal progenitors.

\section{$N t 1$ directly activates cyp26a1}

To determine if $\mathrm{Ntl}$ regulation of cyp26a1 is direct, a Ntlglucocorticoid receptor (Ntl-GR) fusion protein was overexpressed in embryos. This fusion protein enters the nucleus only when the inducer dexamethasone is added (Kolm and Sive 1995). To examine direct regulation, cycloheximide (Chx) was added $30 \mathrm{~min}$ prior to the addition of dexamethasone. In the absence of protein synthesis, the Ntl-GR protein expanded cyp26a1 expression 

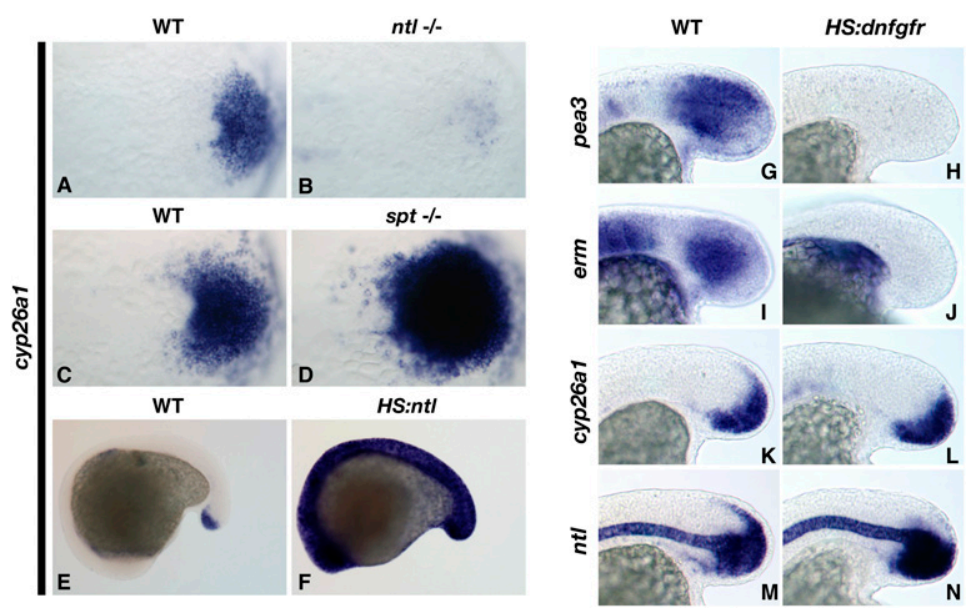

Figure 2. Ntl protects mesodermal progenitors from RA by regulating cyp26a1 expression. $(A-D) \mathrm{Ntl}$ but not Spt is necessary for cyp26a1 expression. cyp26a1 expression is absent in $n t l$ mutants at the eight-somite stage $(B)$ and increased in spt mutants $(D)$ as compared with wild-type siblings $(A, C) .(E, F) \mathrm{Ntl}$ is sufficient for cyp26a1 expression. A HS:ntl transgenic embryo $(F)$ expresses cyp26a1 in all cells $3 \mathrm{~h}$ after heat shock, as opposed to the posteriorly restricted expression in a nontransgenic embryo $(E)$. $(G-N)$ Fgf signaling is not required for cyp26a1 or $n t l$ expression during tailbud stages. HS:dnfgfr embryos completely lack expression of the direct Fgf targets pea3 $(H)$ and erm $(J) 3 \mathrm{~h}$ after heat shock at bud stage compared with normal expression in nontransgenic embryos $(G, I)$, but maintain normal expression of cyp26a1 $(L)$ and $n t l(N)$, as compared with nontransgenic embryos $(K, M)$. $(A-D)$ Dorsal (flat-mounted) view of the posterior end at the eightsomite stage. $(G-N)$ Lateral view of the posterior end at the 16- to 18-somite stage.

only when dexamethasone was added $181 \%$ of Chx/Dextreated embryos hexpanded cyp26a1 expression $[N=27]$, whereas $9 \%$ of embryos treated with $\mathrm{Chx}$ alone had expanded cyp26a1 expression $[N=23])$ (Fig. 3A,B). In order to further substantiate that $\mathrm{Ntl}$ directly activates cyp26a1 expression, we identified potential Ntlbinding sites in the $2.5-\mathrm{kb}$ region upstream of the cyp26a1 transcriptional start site, which has been shown previously to fully recapitulate endogenous cyp26a1 expression (Hu et al. 2008). Using a Ntl position-weighted matrix (Garnett et al. 2009), we identified seven putative Ntl-binding sites with a $P$-value of $<0.001$ (Fig. 3C,E). A luciferase activity assay in $293 \mathrm{~T}$ cells demonstrated that $\mathrm{Ntl}$ is capable of strongly activating the wild-type cyp26a1 promoter (Fig. 3D). A combination of deletions and mutational analysis of individual Ntlbinding sites indicated that the two sites proximal to the cyp26a1 transcriptional start site are essential for the majority of $\mathrm{Ntl}$ activation of the cyp26a1 promoter (Fig. 3D). Combined with the Ntl-GR result, these data indicate that $\mathrm{Ntl}$ acts directly through $\mathrm{Ntl}$-binding sites to activate cyp26a1 expression. The presence of at least five putative Ntl-binding sites with a $P$-value of $<0.001$ within 2.5 $\mathrm{kb}$ of the cyp26a1 transcriptional start site in five different fish species indicates that $\mathrm{Ntl}$ regulation of cyp26a1 is likely evolutionarily conserved (Fig. 3E).

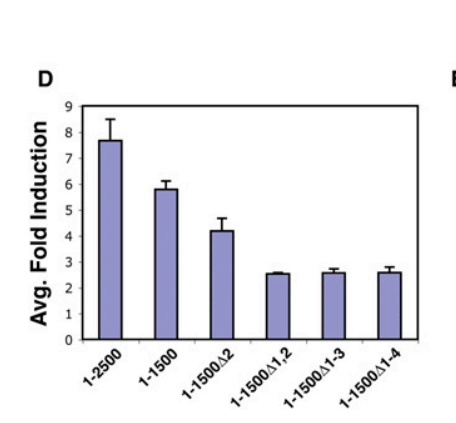

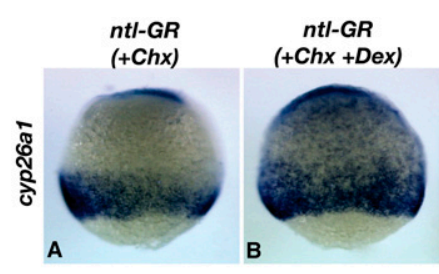

\section{$N t$ establishes the embryonic mesodermal progenitor niche}

In a whole-embryo context, loss of $\mathrm{Ntl}$ results in a loss of wnt3a, wnt8, and cyp26a1 expression (Fig. 2; Martin and Kimelman 2008), causing a failure to sustain the mesodermal progenitors and a subsequent posterior truncation. Surprisingly, we found that the loss of $\mathrm{Ntl}$ function within single mesodermal progenitor cells transplanted into a wild-type environment has no consequence on their development, and they can contribute to somites at all axial levels (Martin and Kimelman 2008). One possibility is that single cells lacking $\mathrm{Ntl}$ are rescued by surrounding wild-type progenitor cells, which provide Wnt ligands and reduce RA levels in the progenitor environment such that any single cell does not need to produce the cytosolic enzyme Cyp26a1 to degrade RA itself. Alternatively, the surrounding wild-type cells could produce a signal that would induce the transcription of the wnts and cyp26a1 in the $n t l$ mutant cells. To distinguish between these two possibilities, ntl morphant cells containing fluorescein dextran were transplanted into wild-type hosts, which were later fixed and examined for the expression of wnt $3 a$, wnt8, and cyp26a1. While control wild-type transplants express these genes normally, $n t l$ morphant cells fail to express all three genes $(80 \%, N=10 ; 90 \%, N=$ 10 ; and $70 \%, N=17$, respectively) (Fig. 4, cf. D-F and $\mathrm{A}-\mathrm{C})$. Therefore, Ntl functions cell-autonomously to activate the wnt genes and cyp26a1 in the progenitor domain.

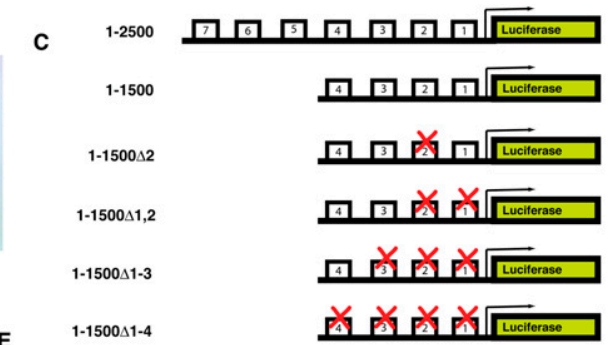

Figure 3. $\mathrm{Ntl}$ directly activates cyp26a1 transcription. $(A, B) \mathrm{Ntl}$ activates cyp26a1 expression in the absence of protein synthesis. Embryos injected with $n t l-G R$ mRNA and treated with Chx $(A)$ show no increase in cyp26a1 expression, whereas treatment with Chx and dexamethasone $(B)$ causes a strong expansion of cyp26a1. (C) Pcyp26a1:1uciferase constructs used for testing $\mathrm{Ntl}$ activation of the cyp26a1 promoter. Boxes 1-7 represent potential Ntlbinding sites ( $E$ shows the true locations of these sites within the promoter). (D) The two most proximal Ntl-binding sites are essential for cyp26a1 induction by $\mathrm{Ntl}$. The graph shows the fold induction of Pcyp26a1:luciferase by $\mathrm{Ntl}$ for each of the constructs shown in $C$. $(E) \mathrm{A}$ minimum of five Ntl-binding sites with a $P$-value of $<0.001$ are found in a 2.5 -kb region upstream of the cyp26a1 transcriptional start site in five different fish species. The numbers above the boxes show the calculated $P$-value. $(A, B)$ Lateral view at $75 \%$ epiboly. 

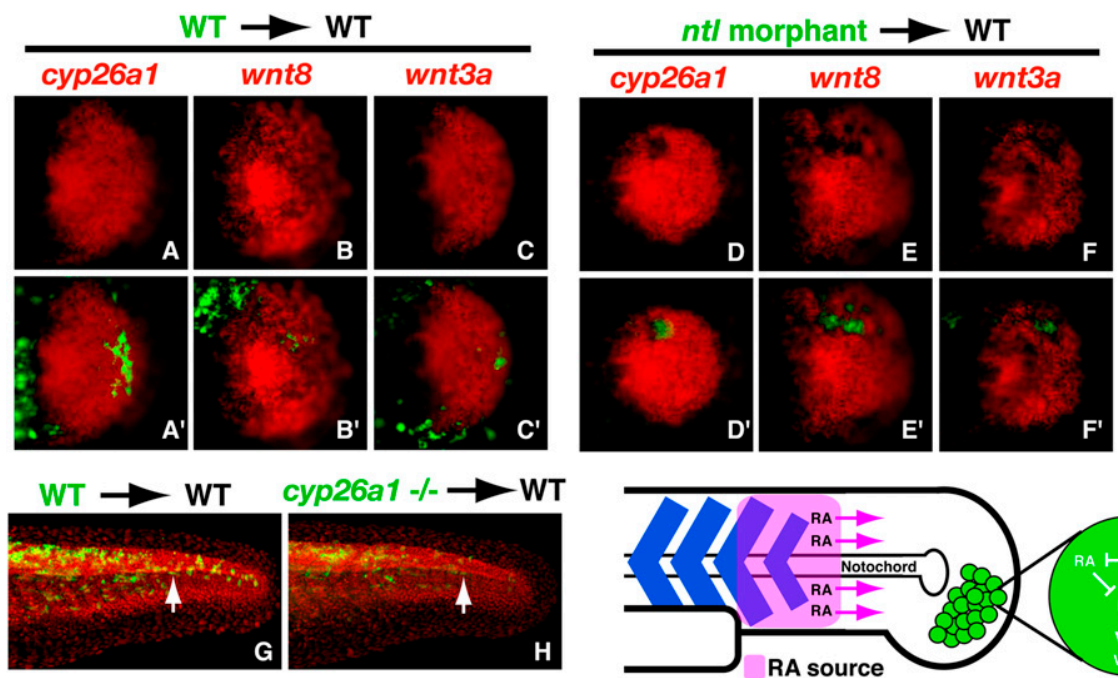

H

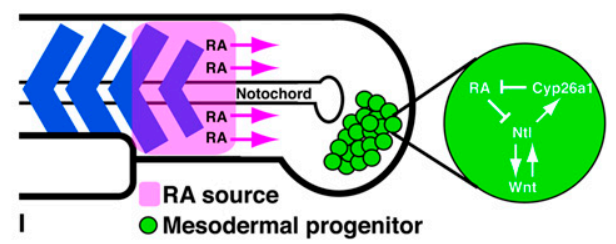

Figure 4. Ntl establishes the mesodermal progenitor niche. $\left(A-F^{\prime}\right) \mathrm{Ntl}$ is cell-autonomously required for the expression of cyp26a1, wnt8, and wnt3a. (A-C') Transplanted wild-type cells have no effect on cyp26a1 $\left(A, A^{\prime}\right)$, wnt8 $\left(B, B^{\prime}\right)$, or wnt3a $\left(C, C^{\prime}\right)$ expression, whereas transplanted nt morphant cells do not express any of the three genes: cyp26a1 $\left(D, D^{\prime}\right)$, wnt8 $\left(E, E^{\prime}\right)$, or wnt $3 a\left(F, F^{\prime}\right)$. The top panels show the in situ hybridization alone (red), whereas the bottom panels are a merge showing the transplanted cells (green) along with the in situ hybridization. (D-F) Note the absence of expression in the top panels in locations where the transplanted $n t l$ morphant cells are found. $(G, H)$ Cyp26al functions non-cellautonomously in mesodermal progenitors. cyp26a1 mutant cells $(H)$ can contribute to the posterior-most somites of wild-type embryos equally as well as wild-type cells $(G)$ in a wild-type embryo (arrows). Transplanted cells are green, whereas all nuclei are labeled in red to show the structure of the embryo. (I) Model of Ntl function in the mesodermal progenitors. Ntl creates the progenitor niche by regulating wnt ligand expression and cyp26a1 expression, which prevents RA produced from neighboring somites from having an effect on the progenitors.

Although cyp26a1 expression was clearly very reduced in $n t l$ morphant cells (Fig. 4D), it remained possible that a low level of cyp26a1 expression could still provide a protective function. We therefore asked if cells lacking all Cyp26al would be able to function normally. Cells from cyp26a1 mutants (giraffe) (Emoto et al. 2005) were transplanted into wild-type hosts and, like $n t l$ mutant transplants, were found to develop normally and populate somites at all axial levels $150 \%$ host embryos with cyp26a1 mutant cells in somites posterior to the 24 th somite, $N=18 ; 52 \%$ host embryos with control wild-type cells in somites posterior to the 24th somite, $N=21$ ) (Fig. 4G,H). Since cyp26a1 mutants have a truncated body (Emoto et al. 2005), we conclude that the mesodermal progenitor population functions as a community to degrade somite-derived RA at the posterior end of the embryo, whereas any individual cell does not have to degrade RA. Combined with our observation that cyp26a1 mutants have reduced $n t l$ expression during the post-gastrula stages (data not shown), we conclude that an essential role of $\mathrm{Ntl}$ is to activate cyp26a1 expression within the community of progenitor cells in order to prevent endogenous RA from repressing $n t l$, which would then suppress the Ntl/Wnt autoregulatory loop and thereby cause truncation of the embryo.

\section{Conclusion}

Common examples of stem or progenitor cell niches in both embryonic and adult organisms consist of at least two general cell types: the stem/progenitor cells and the support cells, which provide the physical and molecular environment necessary for the maintenance of the stem/ progenitor cells (Morrison and Spradling 2008; Voog and Jones 2010). Our data provide evidence of a unique type of progenitor cell niche consisting of only one cell type, in which mesodermal progenitor cells of the zebrafish tailbud act as their own support cells. Mesodermal progenitors express $n t 1, w n t 3 a$, wnt8, and cyp26a1, all of which are required within the progenitor population as a whole, but none of which are required by individual progenitor cells in a wild-type environment. This demonstrates that the wild-type mesodermal progenitor cells act as support cells for the genetically deficient progenitors and can sustain them by creating an environment of high Wnt and low RA signaling. The primary function of Ntl, therefore, is to create the mesodermal progenitor niche through direct regulation of canonical wnt ligands and cyp26a1 (Fig. 4I). While our analysis focused specifically on zebrafish, the common phenotypes of brachyury loss of function and RA treatment in different vertebrates (Dobrovolskaïa-Zavadskaïa 1927; Murakami and Kameyama 1965; Sive et al. 1990; Halpern et al. 1993; Schulte-Merker et al. 1994; Conlon et al. 1996; Martin and Kimelman 2008), as well as the conservation of expression patterns of brachyury, wnts, and cyp26a1, indicates that the same mechanism is common to all vertebrates. Thus, we suggest that expression of brachyury in the progenitor domain was a vertebrate adaptation that allowed the progenitor cells to be sustained during the long process of somitogenesis, which in some species can last for many days (Gomez et al. 2008). This unique function of Brachyury is particularly relevant, given that recent molecular analysis of various human cancers has demonstrated that brachyury is commonly up-regulated in tumors (for example, Vujovic et al. 2006; Palena et al. 2007; Yang et al. 2009; Yoshida et al. 2010). The up-regulation of brachyury may, in effect, be creating a cancer cell niche that maintains high Wnt signaling and low RA signaling, both of which have been extensively 
demonstrated to be key components of cancer growth (Freemantle et al. 2003; Reya and Clevers 2005; Fodde and Brabletz 2007; Osanai et al. 2010).

\section{Materials and methods}

\section{HS:ntl-myc transgenic zebrafish}

myc-tagged ntl (also referred to as ntla) (Goering et al. 2003) was inserted into a previously described vector (Bajoghli et al. 2004) containing a bidirectional heat-shock promoter, GFP, and tol2 transposable elements. Tol2-mediated transgenesis was used to produce a stable transgenic line as described previously (Kawakami 2004). Heat shocks were performed for 30 min at $41^{\circ} \mathrm{C}$ on embryos from an outcross of HS:ntl-myc hemizygous adults; transgenic embryos were separated from nontransgenic embryos based on GFP fluorescence.

\section{HS:dnfgfr1-GFP transgenic zebrafish}

$T g(h s p 70 I: \operatorname{dnfgfr1-EGFP)}$ fish were kindly provided by Ken Poss (Lee et al. 2005). Hemizygous adults were outcrossed and progeny were heatshocked at the 12 -somite stage for $30 \mathrm{~min}$ at $37^{\circ} \mathrm{C}$. Transgenic embryos were sorted based on GFP fluorescence and fixed $3 \mathrm{~h}$ after the end of the 30-min heat shock.

\section{Cell transplantation}

Embryos were injected with $2 \%$ fluorescein dextran either alone,or in combination with the ntl MO (Martin and Kimelman 2008), and were transplanted into the ventral margin of shield stage uninjected WIK/AB host embryos using a CellTram (Eppendorf) (Martin and Kimelman 2008). The same procedure was used for cyp26a1 mutant (giraffe) transplants. Donor embryos were examined at $24 \mathrm{~h}$ post-fertilization to identify homozygous mutants.

\section{RNA injection and in situ hybridization}

The ntl-GR injections and in situ hybridizations were performed as described previously (Martin and Kimelman 2008; Griffin et al. 1995).

\section{cyp26a1 promoter analysis}

The Pcyp26a1-luciferase construct (Hu et al. 2008), which contains $\sim 2500$ base pairs $(\mathrm{bp})$ upstream of the start codon, was kindly provided by Qingshun Zhao. The 1-1500 construct was made by cutting with AgeI (at -1500 ) and the SacI site in the vector, blunting the ends, and religating. Individual sites were mutated using the QuickChange method (Stratagene) to change positions 4 and 5 of the Ntl motif from CA to AT /Garnett et al 2009). The mutagenic oligonucleotides used were (base changes shown in lowercase) site 1, CCACAATTAAAGATGAACTTTGatTGAACTAATTT ATCTGAGGAAGTTAACAGGAGG; site 2, GTGTCGGGGGAATTAAat CCTTTTCAAAGTGAAATCTCAGGATTGTCTGC; site 3, GAGTAGGC TGTCTAACATGatTTATAGCCTTTTAAGGACCCCCAGAGC; and site 4, CATGTATAGAACTTTACATTTATAGAAATATCCAGAAATCAatCTA TTATAATCAACTTTTGAAAATAAAATGCTTTCTATTAT. Luciferase assays used the Dual Luciferase Reporter Assay System (Promega) according to the manufacturer's instructions. 293T cells were transfected with Lipofectamine 2000 (Invitrogen) in a 24-well plate using $50 \mathrm{ng}$ of each Pcyp26a1-luciferase construct, $20 \mathrm{ng}$ of a TK-Renilla luciferase plasmid (Promega), and 150 ng of Ntl-myc expressed from a CMV promoter (Goering et al. 2003) and assayed $48 \mathrm{~h}$ after transfection.

For identification of Ntl-binding sites in cyp26a1 promoters from different fish species, the 2500-bp upstream region was identified from the ENSEMBL database, and the PATSER program (http://rsat.ulb.ac.be/ rsat/patser_form.cgil was used with the Ntl position-weighted matrix (Garnett et al. 2009). Only sites with a $P$-value of $\leq 0.001$ were counted as binding sites.

\section{Acknowledgments}

We are very grateful to Hank Farr for performing the luciferase assays, and we thank Richard Row and Ali Guler for comments on this manuscript,
Joshua Waxman for RAR expression constructs, Ken Poss for the HS:dnfgfr1 line, Qingshun Zhao for the Pcyp26a1-luciferase plasmid, and Cecilia Moens for providing giraffe embryos. This work was supported by an NIH grant (GM079203) to D.K., and an American Cancer Society fellowship (PF-07-048-01-DDC) to B.L.M.

\section{References}

Abu-Abed S, Dolle P, Metzger D, Beckett B, Chambon P, Petkovich M. 2001. The retinoic acid-metabolizing enzyme, CYP26A1, is essential for normal hindbrain patterning, vertebral identity, and development of posterior structures. Genes Dev 15: 226-240.

Bajoghli B, Aghaallaei N, Heimbucher T, Czerny T. 2004. An artificial promoter construct for heat-inducible misexpression during fish embryogenesis. Dev Biol 271: 416-430.

Conlon FL, Sedgwick SG, Weston KM, Smith JC. 1996. Inhibition of Xbra transcription activation causes defects in mesodermal patterning and reveals autoregulation of Xbra in dorsal mesoderm. Development 122: $2427-2435$.

Diez del Corral R, Olivera-Martinez I, Goriely A, Gale E, Maden M, Storey K. 2003. Opposing FGF and retinoid pathways control ventral neural pattern, neuronal differentiation, and segmentation during body axis extension. Neuron 40: 65-79.

Dobbs-McAuliffe B, Zhao Q, Linney E. 2004. Feedback mechanisms regulate retinoic acid production and degradation in the zebrafish embryo. Mech Dev 121: 339-350.

Dobrovolskaïa-Zavadskaïa N. 1927. Sur la mortification spontanee de la chez la souris nouveau-nee et sur l'existence d'un caractere (facteur) hereditaire non-viable. Crit Rev Soc Biol. 97: 114-116.

Dubrulle J, Pourquie O. 2004. Coupling segmentation to axis formation. Development 131: 5783-5793.

Emoto Y, Wada H, Okamoto H, Kudo A, Imai Y. 2005. Retinoic acidmetabolizing enzyme Cyp26a1 is essential for determining territories of hindbrain and spinal cord in zebrafish. Dev Biol 278: 415-427.

Fodde R, Brabletz T. 2007. Wnt/ $\beta$-catenin signaling in cancer stemness and malignant behavior. Curr Opin Cell Biol 19: 150-158.

Freemantle SJ, Spinella MJ, Dmitrovsky E. 2003. Retinoids in cancer therapy and chemoprevention: Promise meets resistance. Oncogene 22: 7305-7315.

Garnett AT, Han TM, Gilchrist MJ, Smith JC, Eisen MB, Wardle FC, Amacher SL. 2009. Identification of direct T-box target genes in the developing zebrafish mesoderm. Development 136: 749-760.

Goering LM, Hoshijima K, Hug B, Bisgrove B, Kispert A, Grunwald DJ. 2003. An interacting network of T-box genes directs gene expression and fate in the zebrafish mesoderm. Proc Nat1 Acad Sci 100: 94109415.

Gomez C, Ozbudak EM, Wunderlich J, Baumann D, Lewis J, Pourquie O. 2008. Control of segment number in vertebrate embryos. Nature 454: 335-339.

Griffin KJ, Kimelman D. 2002. One-Eyed Pinhead and Spadetail are essential for heart and somite formation. Nat Cell Biol 4: 821-825.

Griffin K, Patient R, Holder N. 1995. Analysis of FGF function in normal and no tail zebrafish embryos reveals separate mechanisms for formation of the trunk and the tail. Development 121: 2983-2994.

Halpern ME, Ho RK, Walker C, Kimmel CB. 1993. Induction of muscle pioneers and floor plate is distinguished by the zebrafish no tail mutation. Cell 75: 99-111.

Hu P, Tian M, Bao J, Xing G, Gu X, Gao X, Linney E, Zhao Q. 2008. Retinoid regulation of the zebrafish cyp26a1 promoter. Dev Dyn 237: 3798-3808.

Kawakami K. 2004. Transgenesis and gene trap methods in zebrafish by using the Tol2 transposable element. Methods Cell Biol 77: 201-222.

Kawakami Y, Raya A, Raya RM, Rodriguez-Esteban C, Belmonte JC. 2005. Retinoic acid signalling links left-right asymmetric patterning and bilaterally symmetric somitogenesis in the zebrafish embryo. Nature 435: 165-171.

Kessel M, Gruss P. 1991. Homeotic transformations of murine vertebrae and concomitant alteration of Hox codes induced by retinoic acid. Cell 67: 89-104.

Kolm PJ, Sive HL. 1995. Efficient hormone-inducible protein function in Xenopus laevis. Dev Biol 171: 267-272. 
Lee Y, Grill S, Sanchez A, Murphy-Ryan M, Poss KD. 2005. Fgf signaling instructs position-dependent growth rate during zebrafish fin regeneration. Development 132: 5173-5183.

Martin BL, Kimelman D. 2008. Regulation of canonical Wnt signaling by Brachyury is essential for posterior mesoderm formation. Dev Cell 15: 121-133.

Martin BL, Kimelman D. 2009. Wnt signaling and the evolution of embryonic posterior development. Curr Biol 19: R215-R219. doi: 10.1016/j.cub.2009.01.052.

Morrison SJ, Spradling AC. 2008. Stem cells and niches: Mechanisms that promote stem cell maintenance throughout life. Cell 132: 598-611.

Murakami U, Kameyama Y. 1965. Malformations of the mouse fetus caused by hypervitaminosis-a of the mother during pregnancy. Arch Environ Health 10: 732-741.

Niederreither K, McCaffery P, Drager UC, Chambon P, Dolle P. 1997. Restricted expression and retinoic acid-induced downregulation of the retinaldehyde dehydrogenase type $2(R A L D H-2)$ gene during mouse development. Mech Dev 62: 67-78.

Osanai M, Sawada N, Lee GH. 2010. Oncogenic and cell survival properties of the retinoic acid metabolizing enzyme, CYP26A1. Oncogene 29: 1135-1144.

Palena C, Polev DE, Tsang KY, Fernando RI, Litzinger M, Krukovskaya LL, Baranova AV, Kozlov AP, Schlom J. 2007. The human T-box mesodermal transcription factor Brachyury is a candidate target for T-cell-mediated cancer immunotherapy. Clin Cancer Res 13: 24712478.

Reya T, Clevers H. 2005. Wnt signalling in stem cells and cancer. Nature 434: 843-850.

Sakai Y, Meno C, Fujii H, Nishino J, Shiratori H, Saijoh Y, Rossant J, Hamada H. 2001. The retinoic acid-inactivating enzyme CYP26 is essential for establishing an uneven distribution of retinoic acid along the anterio-posterior axis within the mouse embryo. Genes Dev 15: 213-225.

Schulte-Merker S, van Eeden FJ, Halpern ME, Kimmel CB, NussleinVolhard C. 1994. no tail (nt1) is the zebrafish homologue of the mouse $T$ (Brachyury) gene. Development 120: 1009-1015.

Shum AS, Poon LL, Tang WW, Koide T, Chan BW, Leung YC, Shiroishi T, Copp AJ. 1999. Retinoic acid induces down-regulation of Wnt-3a, apoptosis and diversion of tail bud cells to a neural fate in the mouse embryo. Mech Dev 84: 17-30.

Sirbu IO, Duester G. 2006. Retinoic-acid signalling in node ectoderm and posterior neural plate directs left-right patterning of somitic mesoderm. Nat Cell Biol 8: 271-277.

Sive HL, Draper BW, Harland RM, Weintraub H. 1990. Identification of a retinoic acid-sensitive period during primary axis formation in Xenopus laevis. Genes Dev 4: 932-942.

Thisse C, Thisse B. 2008. Expression from: Unexpected novel relational links uncovered by extensive developmental profiling of nuclear receptor expression. ZFIN direct data submission. http://zfin.org. ZFIN ID: ZDB-PUB-080220-1.

Vermot J, Pourquie O. 2005. Retinoic acid coordinates somitogenesis and left-right patterning in vertebrate embryos. Nature 435: 215-220.

Voog J, Jones DL. 2010. Stem cells and the niche: A dynamic duo. Cell Stem Cell 6: 103-115.

Vujovic S, Henderson S, Presneau N, Odell E, Jacques TS, Tirabosco R, Boshoff C, Flanagan AM. 2006. Brachyury, a crucial regulator of notochordal development, is a novel biomarker for chordomas. I Pathol 209: 157-165.

Wahl MB, Deng C, Lewandoski M, Pourquie O. 2007. FGF signaling acts upstream of the NOTCH and WNT signaling pathways to control segmentation clock oscillations in mouse somitogenesis. Development 134: 4033-4041.

Yang XR, Ng D, Alcorta DA, Liebsch NJ, Sheridan E, Li S, Goldstein AM, Parry DM, Kelley MJ. 2009. T (brachyury) gene duplication confers major susceptibility to familial chordoma. Nat Genet 41: 1176-1178.

Yoshida A, Oda R, Shibahara J, Fukayama M, Tsuda H. 2010. Soft-tissue hemangioblastoma of the retroperitoneum: A case study and review of the literature. Appl Immunohistochem Mol Morphol 18: 479-482. 


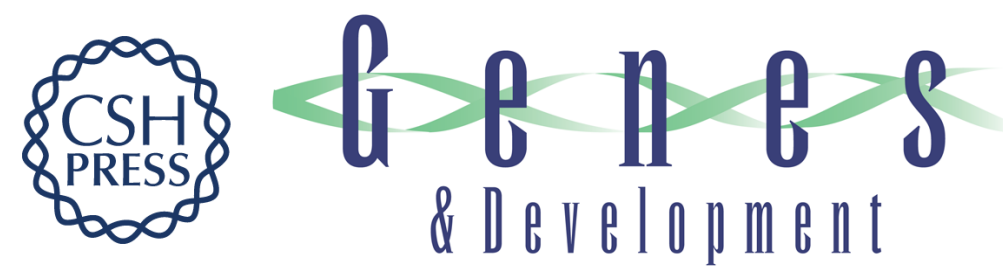

\section{Brachyury establishes the embryonic mesodermal progenitor niche}

Benjamin L. Martin and David Kimelman

Genes Dev. 2010, 24:

Access the most recent version at doi:10.1101/gad.1962910

Supplemental
Material http://genesdev.cshlp.org/content/suppl/2010/12/13/24.24.2778.DC1

References This article cites 41 articles, 12 of which can be accessed free at: http://genesdev.cshlp.org/content/24/24/2778.full.html\#ref-list-1

License

Email Alerting Receive free email alerts when new articles cite this article - sign up in the box at the top Service right corner of the article or click here.

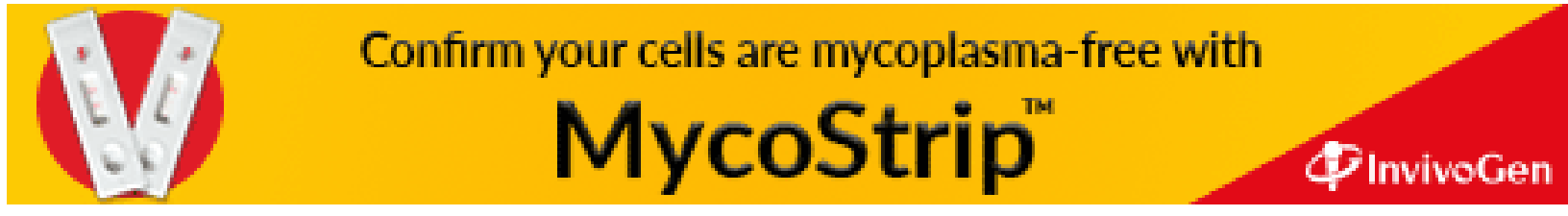

